\title{
An Analytical Solution of a Multi-Winding Coil Problem with a Magnetic Core in Spherical Coordinates
}

\author{
Hüseyin Yıldız ${ }^{1,4 *}$, Erol Uzal², Hüseyin Çalık ${ }^{3}$ \\ ${ }^{1}$ Istanbul University - Cerrahpasa, Department of Mechanical and Metal \\ Technologies, Buyukcekmece, 34500 Istanbul, Turkey, \\ huseyin.yildiz@istanbul.edu.tr \\ ${ }^{2}$ Istanbul University - Cerrahpasa, Department of Mechanical Engineering, \\ Avcilar, 34320 Istanbul, Turkey, euzal@istanbul.edu.tr \\ ${ }^{3}$ Giresun University, Department of Electrical and Electronics Engineering, 28000 \\ Giresun, Turkey, huseyin.calik@giresun.edu.tr \\ ${ }^{4}$ Girift Technology Co., Avcilar, 34320 Istanbul, Turkey
}

\begin{abstract}
Nowadays, technology is advancing rapidly in parallel with developments. The traditional concept of machine loses its function and is replaced by particular devices with spherical geometry such as spherical electric motors and brain stimulation systems. Consequently; the calculation of self-inductance $\left(L_{i i}\right)$ and mutual inductance $\left(M_{i j}\right)$ coefficients in the spherical coordinate system with analytical or semi-analytical methods has become one of the major research topics in recent years. In this study, the terms of $\boldsymbol{B}$, $\boldsymbol{E}$, and $\boldsymbol{A}$ for multi-winding coils were calculated analytically by using the single-winding coil approach. The same geometries were calculated with the assumption of axial symmetry in ANSYS Maxwell using finite element analysis (FEA). The obtained results with the FEA and analytical calculations were compared. Finally, two concentric coil geometries with magnetic core with radius $r_{I}$ were determined, the variation of the self-inductance $\left(L_{i i}\right)$ and mutual inductance $\left(M_{i j}\right)$ coefficients of the determined geometries based on the $\gamma$ angle in spherical coordinates were calculated analytically. Simulation studies were conducted by creating 3D models of coil geometries in ANSYS Maxwell program. An experimental setup that can be produced with 3-dimensional (3D) printers has been designed and constructed compatibly with the determined geometries. The variation of $L_{i i}$ and $M_{i j}$ coefficients with $\gamma$ was studied experimentally after the production of necessary coil windings. At the end of the study, it was observed that the analytical results collected for the mutual inductance $M_{i j}$ and the self-inductance coefficient $L_{i i}$ were consistent with each other by comparing the FEA and experimental results.
\end{abstract}

Keywords: Spherical coordinates; Magnetic field; Maxwell's equations; Analytical solution; Finite element analysis (FEA); Mutual inductance; Self-inductance 


\section{Introduction}

Recently, in parallel with technological developments, the traditional concept of machine has lost its function and replaced with very particular devices. By the advance of accelerator technology, the development of brain stimulation systems, the increase in wireless energy transfer studies, and the development of spherical electric motors, analytical calculation of self-inductance $\left(\mathrm{L}_{\mathrm{ii}}\right)$ and mutual inductance coefficients $\left(\mathrm{M}_{\mathrm{ij}}\right)$ in different coordinate systems has become one of the major research topics. Therefore, the calculation of the inductance coefficient by analytical or semi-analytical methods has attracted great attention in recent years. Much progress has been made in the solution methods in Cartesian and cylindrical coordinates [1-2]. The majority of the studies conducted have an entirely circular geometry. Calculations for circular loops [2-4], cylindrical discs [5], and cylindrical shells [6] can be found in the literature [1]-[7]. Some studies also examine the analytical form of the mutual inductance coefficient for noncoaxial thin and thick coils [8]. These suggested solutions are usually expressed as elliptic integrals, Legendre functions, or Bessel and Struve functions.

In the design of conventional machines, analyses made in Cartesian and cylindrical coordinate systems are sufficient. However, in the analysis of spherical geometry structures such as spherical electric motors and brain stimulation systems, analytical calculation of self-inductance and mutual inductance coefficients in a spherical coordinate system is required. Studies conducted in spherical coordinates have become one of the important research topics in recent years. The first studies on spherical electric motors began in the early 1980s. These studies derived analytical formulas expressing the magnetic field and electric field created by a spherical coil. In a study conducted in 1975, a model with an air core and axial symmetry was addressed. In the study, they obtained a formula giving the magnetic field using the integral transformation method [9]. Another study examined the magnetic field and inductance calculations of an isolated magnetic field analytically generated by a coil having $4 \pi 10^{-7} \mathrm{H} / \mathrm{m}$ magnetic permeability and with diameter $r$ in the outer orbit in a linear and homogeneous isotropic environment [10].

In brain stimulation applications, an analytical method was developed in spherical coordinates to calculate the total electric field in the center caused by the coils placed in various points [11]. The analytical solution of Maxwell's equations in a spherical coordinate system is obtained based on the assumption of axial symmetry. Matute's study has revealed that the method of decomposition of variables is suitable for reaching the analytical solution for resolving some problems addressed in curvilinear coordinates that can be accepted as axially symmetrical [12]. One of the latest analytical studies conducted in the spherical coordinate system was carried out in 2019 to obtain a homogeneous magnetic field. In this study, they observed the sequential arrangement of a sizable number of coils analytically. At the end of the study, it was observed that the sequentially 
arranged coils with a spherical structure were more homogeneous compared to coils of equal size [13]. Most of the studies include the calculations of vector field potential $\mathbf{A}$, magnetic field $\mathbf{B}$, electric field $\mathbf{E}$ and self-inductance coefficient $\mathrm{L}_{\mathrm{ii}}$ and mutual inductance coefficient $\mathrm{M}_{\mathrm{ij}}$ based on the single-winding coil approach. Most of the analytical terms involve Legendre Polynomials or Associated Legendre Polynomials. Analytical formulas for multi-winding coil structures are not yet available in studies conducted in spherical coordinates. So, finite element analysis (FEA) is used to calculate $\mathbf{E}, \mathbf{B}$, and torque $(\tau)$ magnitudes in the applications of electrical machines with spherical geometry. Spherical induction motor studies the most important examples are [14-18]. New results for the analytical calculation of $\mathbf{E}, \mathbf{B}$, and $\mathrm{M}_{\mathrm{ij}}$ sizes in spherical coordinates will contribute to shortening the calculation time and design process.

In this study; Chapter 2, describes the methods used in the general solution of maxwell's equations in spherical coordinates. Chapter 3 presents the analytical solution of field vectors originating from a single-turn magnetic-core coil (i.e. the coil is wound in a magnetic, conductive material, in this case, iron) in a spherical coordinate system. Analytical formulas were obtained for the magnitudes of the vector field potential $\mathbf{A}$, magnetic field $\mathbf{B}$, and electric field $\mathbf{E}$ formed by a singleturn coil with a magnetic core structure in spherical coordinates and the consistency with the analytical formulas derived for the single-turn coil with air core in the literature was examined. Chapter 4, analytical formulas were suggested expressing $\mathbf{A}, \mathbf{E}$, and $\mathbf{B}$ for a multi-winding coil structure with a magnetic core. This was the first time in the literature. By utilizing the Matlab ${ }^{\mathrm{TM}}$ program, numerical values of $\mathbf{A}$ and $\mathbf{B}$ magnitudes on a plane surface were obtained under the assumption of axial symmetry in different coil structures and compared with FEA. In Chapter 5, the interaction problem of two concentric multi-winding coils is examined, and analytical formulas for self-inductance and mutual inductance coefficients are proposed. Chapter 6, an experimental setup was designed by determining the structures of two concentric coils with magnetic core and radius $\mathrm{r}_{1}$. The mutual inductance coefficient of the coil at different angles was calculated by measuring the voltage and current values in the experimental setup. Experimental results, the FEA analysis results, and the results of analytical formulas were compared. In the study, magnetic field $\mathbf{B}$ and electric field $\mathbf{E}$ magnitudes formed by multi-winding coil structures wound on magnetic core were calculated for the first time using a spherical coordinate system. In chapter 7, the results were evaluated.it has been presented that analytical formulas can be used in the preliminary design of various spherical systems, yielding much faster results compared to Three-dimensional FEA analyses. This is important for the analysis of spherical electrical machines. 


\section{General Separated Solution in Spherical Coordinates}

The equations determining the function of electromagnetic systems are called Maxwell's equations. The solution of Maxwell's equations under the conditions of the boundary specific to the problem makes it possible to calculate $\mathbf{B}, \mathbf{E}$, and inductance coefficients. The differential form of Maxwell's equations for electromagnetic systems which consists of linear, isotropic, permeable, and conductive materials is given below [19].

$$
\begin{aligned}
& \nabla \bullet \mathbf{E}=\rho \\
& \text { (Gauss Law) } \\
& \nabla \bullet \mathbf{B}=0 \\
& \nabla \times \mathbf{E}=-\frac{\partial \mathbf{B}}{\partial t} \quad \text { (Faraday Law) } \\
& \nabla \times \mathbf{B}=\mu \mathbf{J}+\mu \mathrm{ò} \frac{\partial \mathbf{E}}{\partial t} \quad \text { (Maxwell Modified Ampère Law) }
\end{aligned}
$$

Here, $\mu$ and ò, are the magnetic permeability constant and the dielectric permeability constant of the field and $\rho$ and $\mathbf{J}$ are the free electric charge density and the current density. $\mathbf{E}$ and $\mathbf{B}$ can be expressed in terms of scalar potential $(\phi)$ and vector potential (A), as follows [20].

$$
\nabla^{2} \phi=0
$$

The terms of $\mathbf{E}$ and $\mathbf{B}$ are given below as the type of vector $\mathbf{A}$ [21].

$$
\begin{aligned}
& \mathbf{E}=-i \omega \mathbf{A} \\
& \mathbf{B}=\nabla \times \mathbf{A} \\
& \nabla^{2} \mathbf{A}-\mu \grave{\mathrm{o}} \frac{\partial^{2} \mathbf{A}}{\partial t^{2}}=-\mu \mathbf{J}
\end{aligned}
$$

Here, $\mathrm{i}$ and $\omega$ indicate $90^{\circ}$ degrees phase angle and angular frequency (R/s) respectively. The solution of Eq. (5) in the spherical coordinate system provides the representation of the vector potential in spherical coordinates. Since no current flows except the $r_{0}, \theta_{0}$ coordinates in which the coil exists, we can write $\mathbf{J}=0$ outside the coil.

$$
\nabla^{2} \mathbf{A}-\mu \grave{\mathrm{o}} \frac{\partial^{2} \mathbf{A}}{\partial t^{2}}=0
$$


The homogeneous partial differential equation given by Eq. (6) is the electromagnetic wave equation, and $\mathbf{A}$ in linear systems with time oscillations can be written in the form below [12].

$$
\mathbf{A}(r, \theta, t)=\mathbf{A}_{\varphi}(r, \theta) e^{-i \omega t}
$$

If $\mathbf{A}$ as the term of vector field potential given by Eq. (7) is written in Eq. (6), the vector Helmholtz equation is found. The vector Helmholtz equation allows us to find the solution to the problem based on the boundary conditions regardless of time. If $\mathrm{k}^{2}<<1$ when solving the Helmholtz equation, the term $k^{2}=\omega \mu \sigma$ is ignored.

$\nabla^{2} \mathbf{A}=0$

If $\mathbf{A}_{\mathbf{0}}=A_{0}(r, \theta) \mathbf{e}_{\varphi}$ based on the assumption of axial symmetry, then the expanded form of Eq. (8) is found as Eq. (9) in spherical coordinates.

$\nabla^{2} \mathbf{A} \varphi=\frac{1}{r} \frac{\partial^{2}\left(r A_{\varphi}\right)}{\partial r^{2}}+\frac{1}{r^{2}} \frac{\partial^{2} A_{\varphi}}{\partial \theta^{2}}+\frac{\cot \theta}{r^{2}} \frac{\partial A_{\varphi}}{\partial \theta}-\frac{1}{r^{2}} \frac{A_{\varphi}}{\sin ^{2} \theta}$

If the partial differential equation given by Eq. (9) is solved with the method of the decomposition of the variables, the serial solution of $\mathbf{A}$ is obtained. Here, the term $P_{n}^{1}(\cos \theta)$ represents the $1^{\text {st }}$ order Associated Legendre Polynomial. The equation given by Eq. (10) is expressed as the general separated solution in the spherical coordinate system [22] and so on.

$$
A_{\varphi}=\sum_{1}^{\infty}\left(a_{n} r^{n}+b_{n} r^{-n-1}\right) P_{n}^{1}(\cos \theta)
$$

\section{Calculation of Magnetic Field and Electric Field for a Single-Winding Coil with Magnetic Core}

In spherical electrical machines, rotor and stator windings are wound around a magnetic core. So, analyzes must be made in the spherical coordinate system. A spherical electric machine in its simplest form consists of a single winding coil structure wound on a magnetic core. In real applications, there are multi-winding coil structures on the magnetic core. For the first problem, the definitions and geometric features required in the solution of the problem are defined in spherical coordinates and shown in Figure 1.

Figure 1a shows the magnetic sphere and the coil winding around it. The coil winding is wound outside the sphere structure and it is symmetrical concerning the z-axis. In Figure 1b, the coil structure is placed on the x-y plane. The coil is 
placed in the $r_{0}$ and $\theta_{0}$ coordinates in the spherical coordinates. By monitoring the electric and magnetic field components of the coil on three different fields of $A_{0}$, $A_{1}$ and $A_{2}$, the representation of the vector field potential within these fields will be calculated.

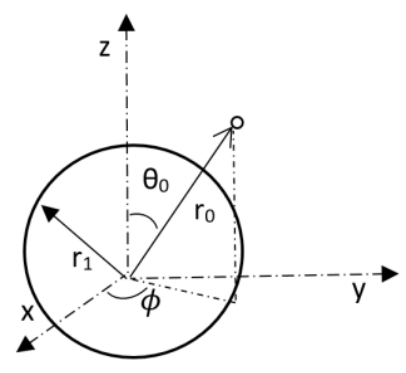

$b$

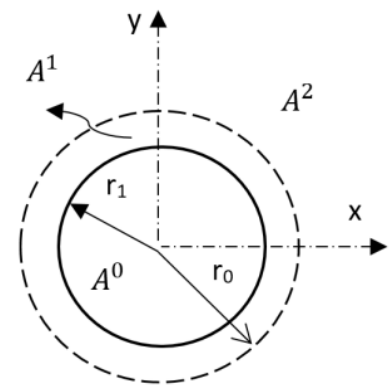

Figure 1

The cross-section view of the sphere and the winding (a)

The top view of the sphere and the winding (b)

$\mathrm{A}^{(0)}, \mathrm{A}^{(1)}$, and $\mathrm{A}^{(2)}$ are the spherical cores, the gap regions inside and outside the radius $r_{0}$ in which the coil is wound, respectively. Where $r$ and $\theta$ are the coordinates of the calculated point, $\mathrm{r}_{1}$ is the radius of the spherical core geometry, $r_{0}$ and $\theta_{0}$ are the coil coordinates, $\mu_{1}$ and $\mu_{0}$ are the magnetic permeability coefficients of the calculation points, and $\mu=\mu_{1} / \mu_{0}$ is the respective magnetic permeability coefficient. Consider region $\mathrm{A}^{(0)}\left(\mathrm{r}<\mathrm{r}_{1}\right)$, the second term in Eq. (10) is neglected because it is divergent. Consider region $\mathrm{A}^{(1)}\left(\mathrm{r}_{1}<\mathrm{r} \leq \mathrm{r}_{0}\right)$, both two terms are used together. Consider region $\mathrm{A}^{(2)}\left(\mathrm{r} \geq \mathrm{r}_{0}\right)$, the first term in Eq. (10) is neglected because it is divergent. In this case, $\mathbf{A}$ is written separately for each of the three regions as follows;

$$
\begin{aligned}
& A^{(0)}(r, \theta)=\sum_{1}^{\infty} a_{n} r^{n} P_{n}^{1}(\cos \theta) \\
& A^{(1)}(r, \theta)=\sum_{1}^{\infty}\left(b_{n} r^{n}+c_{n} r^{-n-1}\right) P_{n}^{1}(\cos \theta) \\
& A^{(2)}(r, \theta)=\sum_{1}^{\infty} d_{n} r^{-n-1} P_{n}^{1}(\cos \theta)
\end{aligned}
$$

Maxwell's equations are valid for all points where physical parameters are constant. However, in some cases, physical parameters may vary such as geometry, current, $\mu$, ò etc. In these cases, the boundary conditions given in Eq. (12) become valid [23]. 
$\mathbf{n} \times\left(\mathbf{E}_{2}-\mathbf{E}_{1}\right)=0$
$\mathbf{n} \times\left(\mathbf{H}_{2}-\mathbf{H}_{1}\right)=\mathbf{J}$
$\left(\mathbf{B}_{2}-\mathbf{B}_{1}\right) \cdot \mathbf{n}=0$
$\left(\mathbf{D}_{2}-\mathbf{D}_{1}\right) \cdot \mathbf{n}=\rho_{s}$

Here $\mathbf{D}$ is the electric current density and $\rho_{s}$ is the surface charge density, if the conductivity values of the two substances are finite, the surface current density $\mathbf{J}=0$, and equations can be rearranged.

$\mathbf{n} \times\left(\mathbf{H}_{2}-\mathbf{H}_{1}\right)=0$

In the coordinates of $r=r_{1}$ and $r=r_{0}$, there is no electric field change in the direction of the surface normal. Since no current occurs on the sphere surface, $\mathbf{J}=0$. On the surface where the winding is $\left(\theta=\theta_{0} r=r_{0}\right), \mathbf{J}$ is non-zero. If it is solved along with the representative solutions given by Eq. (1), four equations with four unknowns are obtained (14).

$$
\begin{aligned}
& \left(b_{n}-a_{n}\right) r_{1}^{n}+c_{n} r_{1}^{-n-1}=0 \\
& b_{n} r_{0}^{n}+\left(c_{n}+d_{n}\right) r_{1}^{n}+c_{n} r_{1}^{-n-1}=0 \\
& (n+1)\left(\frac{1}{\mu} a_{n}-b_{n}\right) r_{1}^{n-1}+n c_{n} r_{1}^{-n-2}=0 \\
& (n+1) b_{n} r_{0}^{n}+n\left(d_{n}-c_{n}\right) r_{0}^{-n-1}-\frac{2 n+1}{2 n(n+1)} I \mu_{0} P_{n}^{1}\left(\cos \theta_{0}\right) \cos \theta_{0}=0
\end{aligned}
$$

The obtained common solution to equations of Eq. (14), gives the coefficients of $a_{n}, b_{n}, c_{n}$, and $d_{n}$ presented in Eq. (15). Using Eq. (11) and Eq. (15), A created by a single-winding coil that is wound on the magnetic core can be calculated.

$$
\begin{aligned}
& a_{n}=\frac{(2 n+1) \mu}{2 n(n+1)(1+n+n \mu)} I \mu_{0} r_{0}^{-n} \sin \theta_{0} P_{n}^{1}\left(\cos \theta_{0}\right) \\
& b_{n}=\frac{1}{2 n(n+1)} I \mu_{0} r_{0}^{-n} \sin \theta_{0} P_{n}^{1}\left(\cos \theta_{0}\right), \\
& c_{n}=\frac{(\mu-1)}{2 n(1+n+n \mu)} I \mu_{0} r_{0}^{-n} r_{1}^{1+2 n} \sin \theta_{0} P_{n}^{1}\left(\cos \theta_{0}\right) \\
& d_{n}=\frac{I \mu_{0} \sin \theta_{0} P_{n}^{1}\left(\cos \theta_{0}\right) r_{0}^{-n}}{2 n(n+1)(1+n+n \mu)}\left((1+n+n \mu) r_{0}^{1+2 n}+(1+n)(\mu-1) r_{1}^{1+2 n}\right)
\end{aligned}
$$

$\mathbf{E}$ and $\mathbf{B}$ is calculated with the help of Eq. (16) depending on A. The expanded form of $\mathbf{B}$ is presented in Eq. (17). 


$$
\begin{aligned}
& \mathbf{E}=-i \omega \mathbf{A} \\
& \mathbf{B}=\nabla \times \mathbf{A} \\
& \mathbf{B}=\frac{1}{r \sin \theta} \frac{\partial}{\partial \theta}\left(A_{\varphi} \sin \theta\right) \mathbf{e}_{\mathbf{r}}-\frac{1}{r} \frac{\partial}{\partial r}\left(r A_{\varphi}\right) \mathbf{e}_{\theta} \\
& \mathbf{B}^{(0)}=\sum_{1}^{\infty} a_{n} r^{n-1} n(n+1) P_{n}(\cos \theta) \mathbf{e}_{r}-\sum_{1}^{\infty}(n+1) a_{n} r^{n-1} P_{n}^{1}(\cos \theta) \mathbf{e}_{\theta} \\
& \mathbf{B}^{(1)}=\sum_{1}^{\infty}\left(b_{n} r^{n-1}+c_{n} r^{-n-2}\right) n(n+1) P_{n}(\cos \theta) \mathbf{e}_{r}-\sum_{1}^{\infty}\left((n+1) b_{n} r^{n-1}-n c_{n} r^{-n-2}\right) P_{n}^{1}(\cos \theta) \mathbf{e}_{\theta} \\
& \mathbf{B}^{(2)}=\sum_{1}^{\infty} n(n+1) d_{n} r^{-n-2} P_{n}(\cos \theta) \mathbf{e}_{r}+\sum_{1}^{\infty} n d_{n} r^{-n-2} P_{n}^{1}(\cos \theta) \mathbf{e}_{\theta}
\end{aligned}
$$

Eq. (17) gives the general solution for the core structures with different magnetic permeability. In Eq. (17) the particular case of $\mu=1$ gives the particular case of the analysis of a coil structure with an air core in spherical coordinates. When Eq. (16) and Eq. (17) are analyzed, it is seen that they are compatible with the equations given in the literature [22].

\section{Calculation of the Integral Form for a Multi- Winding Coil with a Magnetic Core}

By Eq. (17), the analytical form of $\mathbf{B}$ is presented for a single-winding coil wound on a sphere having a magnetic permeability of $\mu_{1}$ and a radius of $r_{1}$. However, in practice, coil windings are wound in multiple windings to include a certain region (Figure 2). For the regions of $A_{0}, A_{1}$ and $A_{2}$, the integral form of $\mathbf{A}$ formed by a multi-winding coil is given in Eq. (18). Where $r_{a}, r_{b}, \theta_{a}$, and $\theta_{b}$ are the starting and ending coordinates of the coil winding, $\mathrm{N}$ is the number of the coil winding, and $\mathrm{S}$ refers to the surface field of the coil winding (Figure 2).

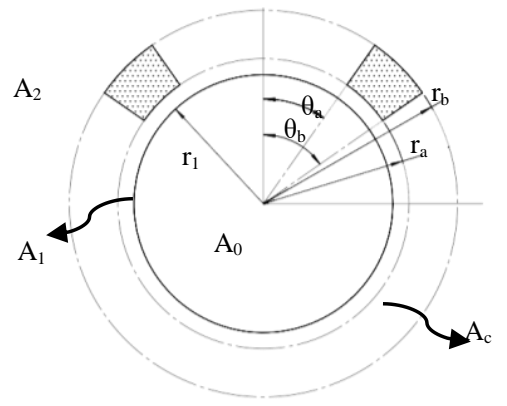

Figure 2

The structure of the multi-winding coil 


$$
\begin{aligned}
& A_{i}^{(0)}(r, \theta)=\int_{r_{a}}^{r_{b} \theta_{\theta_{a}}} A^{(0)}(r, \theta) r_{0} d r_{0} d \theta_{0} \\
& A_{i}^{(1)}(r, \theta)=\int_{r_{a}}^{r_{b} \theta_{b}} \int_{\theta_{a}} A^{(1)}(r, \theta) r_{0} d r_{0} d \theta_{0} \\
& A_{i}^{(2)}(r, \theta)=\int_{r_{a}}^{r_{b_{b}} \theta_{b}} \int_{\theta_{a}} A^{(2)}(r, \theta) r_{0} d r_{0} d \theta_{0} \\
& R=\frac{1}{2}\left(r_{b}^{2}-r_{a}^{2}\right)\left(\theta_{b}-\theta_{a}\right) \\
& R(u, v)=\left\{\begin{array}{cc}
v-u \\
n=1, & \ln \left(\frac{v}{u}\right) \\
n=2, & \frac{1}{2-n}\left(v^{2-n}-u^{2-n}\right)
\end{array}\right\}
\end{aligned}
$$

By the principle of superposition, if the integral is taken on the coefficients of $a_{n}$, $b_{n}, c_{n}$, and $d_{n}$ given in Eq. (15), and on the boundaries of $r_{a}, r_{b}, \theta_{a}$, and $\theta_{b}$ the coefficients representing the structure of a multi-winding coil is obtained.

$$
\begin{aligned}
& a_{n}^{i}=\frac{N(2 n+1) \mu I \mu_{0}}{S 2 n(n+1)(1+n+n \mu)} R\left(r_{a}, r_{b}\right) \int_{\theta_{a}}^{\theta_{b}} \sin \theta_{0} P_{n}^{1}\left(\cos \theta_{0}\right) d \theta_{0} \\
& b_{n}^{i}=\frac{N I \mu_{0}}{S 2 n(n+1)} R\left(r_{a}, r_{b}\right) \int_{\theta_{a}}^{\theta_{b}} \sin \theta_{0} P_{n}^{1}\left(\cos \theta_{0}\right) d \theta_{0} \\
& c_{n}^{i}=\frac{N(\mu-1) I \mu_{0}}{S 2 n(1+n+n \mu)} r_{1}^{1+2 n} R\left(r_{a}, r_{b}\right) \int_{\theta_{a}}^{\theta_{b}} \sin \theta_{0} P_{n}^{1}\left(\cos \theta_{0}\right) d \theta_{0} \\
& d_{n}^{i}=\frac{N(\mu-1) I \mu_{0}}{S 2 n(n+1)(1+n+n \mu)}\left(\begin{array}{l}
\left(\frac{(1+n+n \mu)}{n+3}\left(r_{b}^{3+n}-r_{a}^{3+n}\right)\right) \\
+(1+n)(\mu-1) r_{1}^{1+2 n} R\left(r_{a}, r_{b}\right)
\end{array}\right) \int_{\theta_{a}}^{\theta_{b}} \sin \theta_{0} P_{n}^{1}\left(\cos \theta_{0}\right) d \theta_{0}
\end{aligned}
$$

Assume that the region with the coil winding is divided into two parts from the radius of $\mathrm{r}_{0}$ to calculate the effect of multi-winding in the region of $\mathrm{A}_{\mathrm{c}}\left(r_{a} \leq \mathrm{r}\right.$ $\left.\leq r_{b}\right)$. The coils that need to be calculated in the range of $r_{a}<\mathrm{r}$ and in the $\mathrm{A}_{\mathrm{c}}(\mathrm{r}, \theta)$ region, perform as in the $\mathrm{A}_{2}$ region, and the coils in the range of $\mathrm{r} \leq r_{b}$ perform as in the $A_{1}$ region. In this case, the total vector field potential formed by two regions 
helps to calculate the magnitudes of $\mathbf{A}, \mathbf{E}$, and $\mathbf{B}$ approximately for this region. This assumption gives better results in case the value of $\square r=r_{b}-r_{a}$ is smaller.

$$
\begin{aligned}
& A_{i}^{(c)}(r, \theta)=\int_{r_{a}}^{r} \int_{\theta_{a}}^{\theta_{b}} A^{(2)}(r, \theta) r_{0} d r_{0} d \theta_{0}+\int_{r}^{r_{b}} \int_{\theta_{a}}^{\theta_{b}} A^{(1)}(r, \theta) r_{0} d r_{0} d \theta_{0} \\
& b_{n}^{c}=\frac{N I \mu_{0}}{S 2 n(n+1)} R\left(r, r_{a}\right) \int_{\theta_{a}}^{\theta_{b}} \sin \theta_{0} P_{n}^{1}\left(\cos \theta_{0}\right) d \theta_{0} \\
& c_{n}^{c}=\frac{N(\mu-1) I \mu_{0}}{S 2 n(1+n+n \mu)} r_{1}^{1+2 n} R\left(r, r_{a}\right) \int_{\theta_{a}}^{\theta_{b}} \sin \theta_{0} P_{n}^{1}\left(\cos \theta_{0}\right) d \theta_{0} \\
& \left.d_{n}^{c}=\frac{N(\mu-1) I \mu_{0}}{S 2 n(n+1)(1+n+n \mu)}\left(\begin{array}{l}
\left(\frac{(1+n+n \mu)}{n+3}\left(r_{b}^{3+n}-r^{3+n}\right)\right. \\
+(1+n)(\mu-1) r_{1}^{1+2 n} R\left(r, r_{a}\right)
\end{array}\right)\right) \int_{\theta_{a}}^{\theta_{b}} \sin \theta_{0} P_{n}^{1}\left(\cos \theta_{0}\right) d \theta_{0} \\
& A_{i}^{(0)}(r, \theta)=\sum_{1}^{\infty} a_{n}^{i} r^{n} P_{n}^{1}(\cos \theta) \\
& A_{i}^{(1)}(r, \theta)=\sum_{1}^{\infty}\left(b_{n}^{i} r^{n}+c_{n}^{i} r^{-n-1}\right) P_{n}^{1}(\cos \theta) \\
& A_{i}^{(2)}(r, \theta)=\sum_{1}^{\infty} d_{n}^{i} r^{-n-1} P_{n}^{1}(\cos \theta) \\
& A_{i}^{(c)}(r, \theta)=\sum_{1}^{\infty}\left(b_{n}^{c} r^{n}+\left(c_{n}^{c}+d_{n}^{c}\right) r^{-n-1}\right) P_{n}^{1}(\cos \theta) \\
& \mathbf{B}_{i}^{(0)}=\sum_{1}^{\infty} a_{n}^{i} r^{n-1} n(n+1) P_{n}(\cos \theta) \mathbf{e}_{r}-\sum_{1}^{\infty}(n+1) a_{n}^{i} r^{n-1} P_{n}^{1}(\cos \theta) \mathbf{e}_{\theta} \\
& \mathbf{B}_{i}^{(1)}=\sum_{1}^{\infty}\left(b_{n}^{i} r^{n-1}+c_{n}^{i} r^{-n-2}\right) n(n+1) P_{n}(\cos \theta) \mathbf{e}_{r} \\
& -\sum_{1}^{\infty}\left((n+1) b_{n}^{i} r^{n-1}-n c_{n}^{i} r^{-n-2}\right) P_{n}^{1}(\cos \theta) \mathbf{e}_{\theta} \\
& \mathbf{B}_{i}^{(2)}=\sum_{1}^{\infty} n(n+1) d_{n}^{i} r^{-n-2} P_{n}(\cos \theta) \mathbf{e}_{r}+\sum_{1}^{\infty} n d_{n}^{i} r^{-n-2} P_{n}^{1}(\cos \theta) \mathbf{e}_{\theta} \\
& \mathbf{B}_{i}^{(c)}=\sum_{1}^{\infty}\left(b_{n}^{c} r^{n-1}+\left(c_{n}^{c}+d_{n}^{c}\right) r^{-n-2}\right) n(n+1) P_{n}(\cos \theta) \mathbf{e}_{r} \\
& -\sum_{1}^{\infty}\left((n+1) b_{n}^{c} r^{n-1}-n\left(c_{n}^{c}-d_{n}^{c}\right) r^{-n-2}\right) P_{n}^{1}(\cos \theta) \mathbf{e}_{\theta}
\end{aligned}
$$


The coefficients presented in Eq. (19) and Eq. (20) are used to calculate the magnitudes of $\mathbf{A}$ and $\mathbf{B}$ given in Eq. (21) and Eq. (22). Figure 3 shows how to calculate A and B. With the help of the Matlab program, the analysis parameters given in Table 1 are calculated using the analytical formulas given in Eq. (21) and Eq. (22). The obtained numerical results are given in Figure 4 and Figure 5.

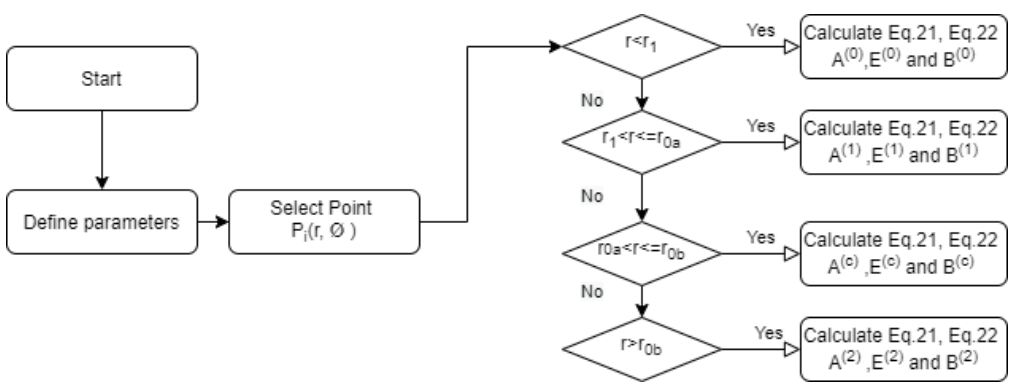

Figure 3

A, $\mathbf{E}$ and $\mathbf{B}$ calculation flowchart

Table 1

The analysis parameters

\begin{tabular}{cl} 
Parameter & \multicolumn{1}{c}{ Value } \\
\hline $\mathrm{I}$ & $1 \mathrm{~A}$ \\
\hline$\mu_{0}(H / m)$ & $4 \pi 10^{-7}$ \\
\hline$\mu(H / m)$ & $1,10,100$ \\
\hline$r_{0}(\mathrm{~mm})$ & 34 \\
\hline$r_{1}(\mathrm{~mm})$ & 30 \\
\hline$r(\mathrm{~mm})$ & $0<r<50$ \\
\hline
\end{tabular}

\begin{tabular}{cl} 
Parameter & Value \\
\hline$\theta_{0}$ & $45^{\circ}$ \\
\hline$\Delta \theta$ & $10^{\circ}$ \\
\hline$\theta$ & $22.5^{\circ}, 30^{\circ}, 45^{\circ}$ \\
\hline $\mathrm{N}$ & 100 \\
\hline & \\
\hline
\end{tabular}

Figure 4 shows the variation with $\mu$ of $\mathbf{B}$ and $\mathbf{A}$ on a sphere of radius $r_{1}$ of a coil placed in $r_{a}, r_{b}, \theta_{a}$, and $\theta_{b}$ coordinates. The values of $\mathbf{A}$ in regions far from the coil winding have small and smooth transitions. Where $\theta=45^{\circ}$ is the direction in which the magnetic effects are the highest. Thus, the graphic in Figure $4 \mathrm{f}$ is the one that the magnetic effect can be observed most clearly. It is obvious that as $\mu$ gets larger, magnetic effects accumulate on the surface of the sphere. 


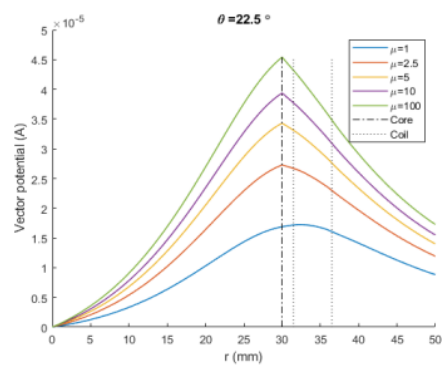

a
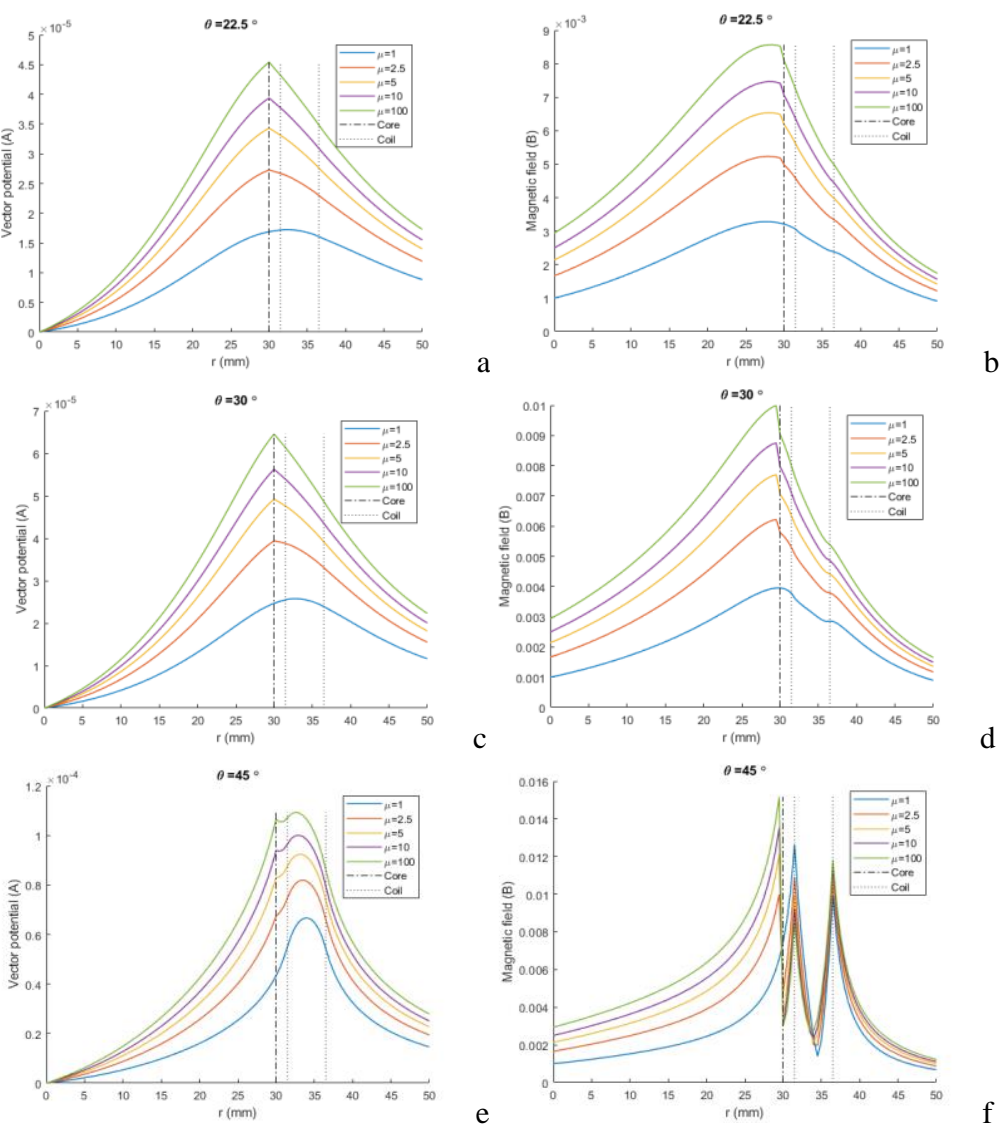

Figure 4

For $\theta=22.5^{\circ}$ the graphic view of the variation of $\mathbf{A}$ for different $\mu$ values (a). For $\theta=22.5^{\circ}$ the graphic view of the variation of $\mathbf{B}$ for different $\mu$ values (b). For $\theta=30^{\circ}$ the graphic view of the variation of $\mathbf{A}$ for different $\mu$ values (c). For $\theta=30^{\circ}$ the graphic view of the variation of $\mathbf{B}$ for different $\mu$ values (d). For $\theta=45^{\circ}$ the graphic view of the variation of $\mathbf{A}$ for different $\mu$ values (e). For $\theta=45^{\circ}$ the graphic view of the variation of $\mathbf{B}$ for different $\mu$ values (f).

Figure 5, shows the variations of $\mathbf{B}$ and $\mathbf{A}$ on the $\mathrm{x}-\mathrm{z}$ plane with the different $\mu$ values. When Figure 4 and Figure 5 are examined, it is witnessed that as $\mu$ increases, the magnitudes of the magnetic field and the vector potential come closer to the sphere surface.

The coil and sphere geometries, the features of which given in Table 1 with the ANSYS Maxwell program were formed under the assumption of 2D axial symmetry. As the magnetostatic analysis, three different analyses were performed as $\mu=1, \mu=10$, and $\mu=100$. 

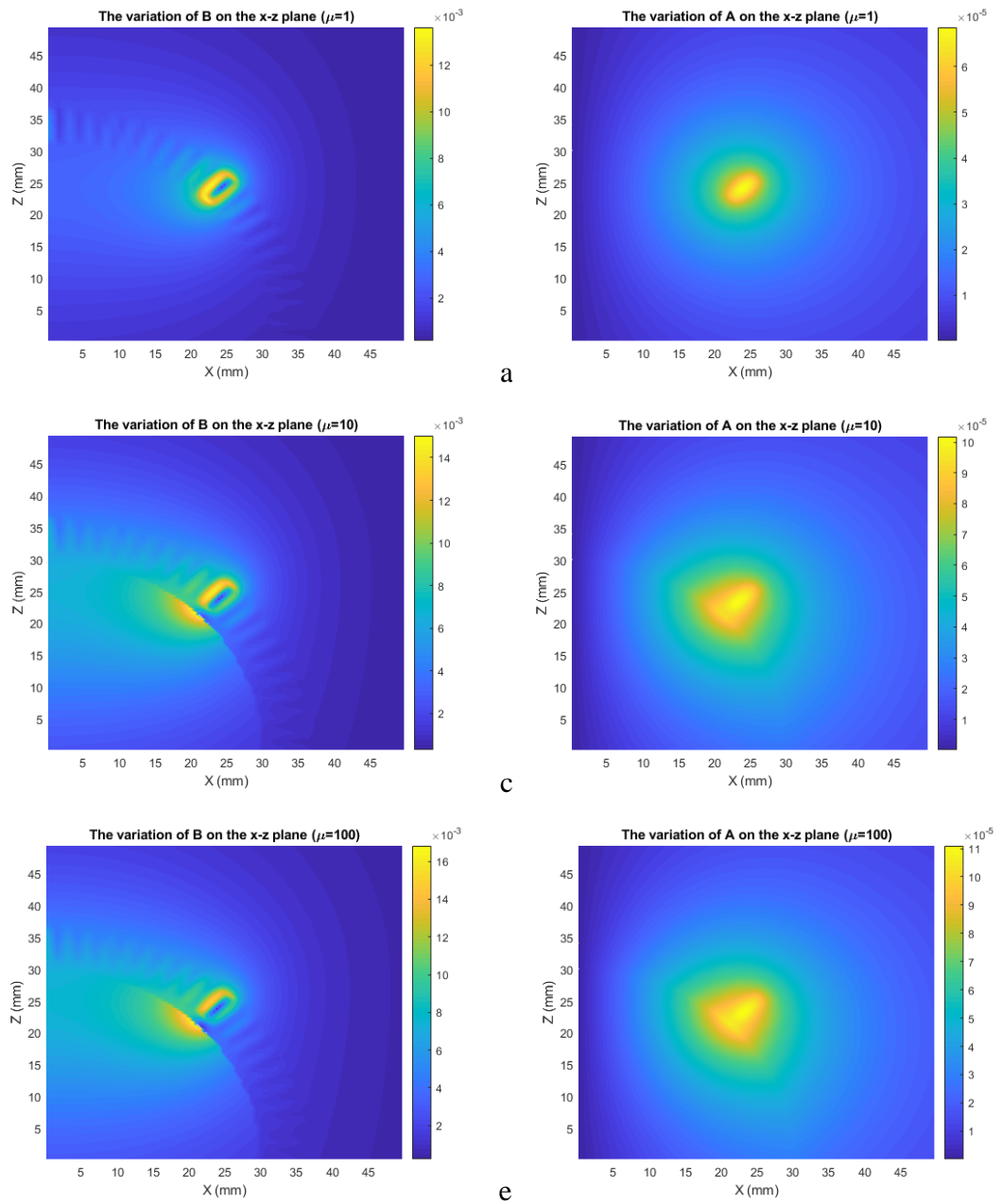

Figure 5

For $\mu=1$, the variation of $\mathbf{B}$ on the $\mathrm{x}-\mathrm{z}$ plane (a). For $\mu=1$, the variation of $\mathbf{A}$ on the $\mathrm{x}-\mathrm{z}$ plane (b). For $\mu=10$, the variation of $\mathbf{B}$ on the $\mathrm{x}-\mathrm{z}$ plane (c). For $\mu=10$, the variation of $\mathbf{A}$ on the $\mathrm{x}-\mathrm{z}$ plane (d). For $\mu=100$, the variation of $\mathbf{B}$ on the $\mathrm{x}-\mathrm{z}$ plane (e). For $\mu=100$, the variation of $\mathbf{A}$ on the $\mathrm{x}-\mathrm{z}$ plane (f).

The distribution of the magnitude of the magnetic field $\mathbf{B}$ on the $\mathrm{x}-\mathrm{z}$ plane which was obtained with the ANSYS Maxwell program is presented in Figure 6. When Figure 6 and Figure 5 are observed, it is seen that the FEA results and the distribution of the magnetic field obtained from analytical calculations give similar results. 

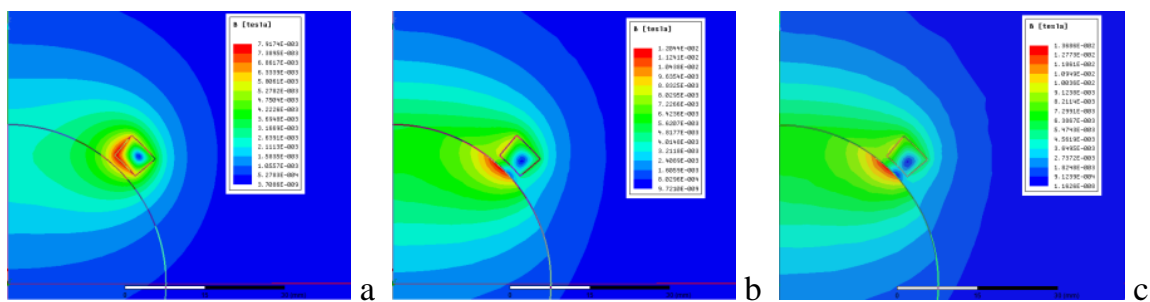

Figure 6

For ANSYS Maxwell $\mu=1$, the distribution of the magnetic field on the X-z plane (a). For ANSYS Maxwell $\mu=10$, the distribution of the magnetic field on the X-Z plane (b). For ANSYS Maxwell $\mu=100$, the distribution of the magnetic field on the $x-z$ plane (c).

In Figure 7 for $\theta=30^{\circ}$ and $\theta=45^{\circ}$ the variation of $\mathbf{B}$ on $r$ is compared with the FEA results. When the results are assessed, it is seen that the magnitudes of the magnetic field obtained with analytical formulas are consistent with the ones obtained from the model of the FEA. The differences in the results become apparent around the coil geometry. In this case, in the calculation of multiwinding coil results, the formula used for the region $A_{c}$ among the coil windings is valid for small values of $r_{b}-r_{a}$. When the distance between coil geometries expands, the differences increase due to the single-coil approach.
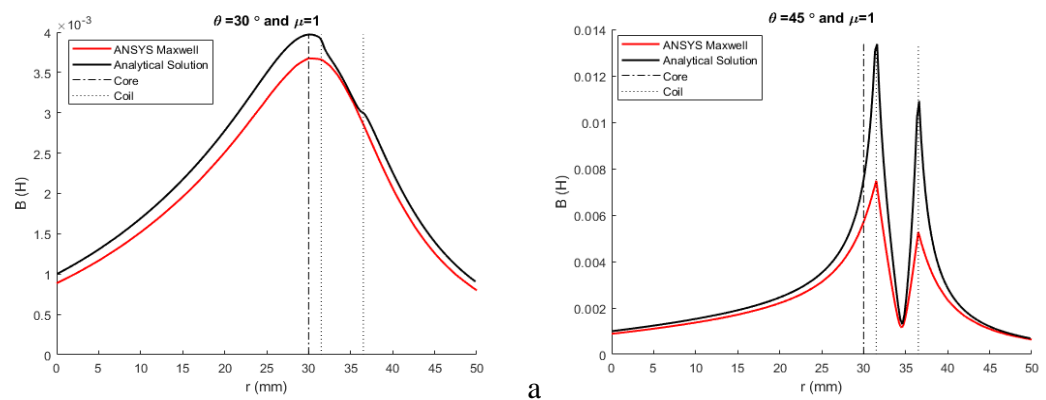

b 

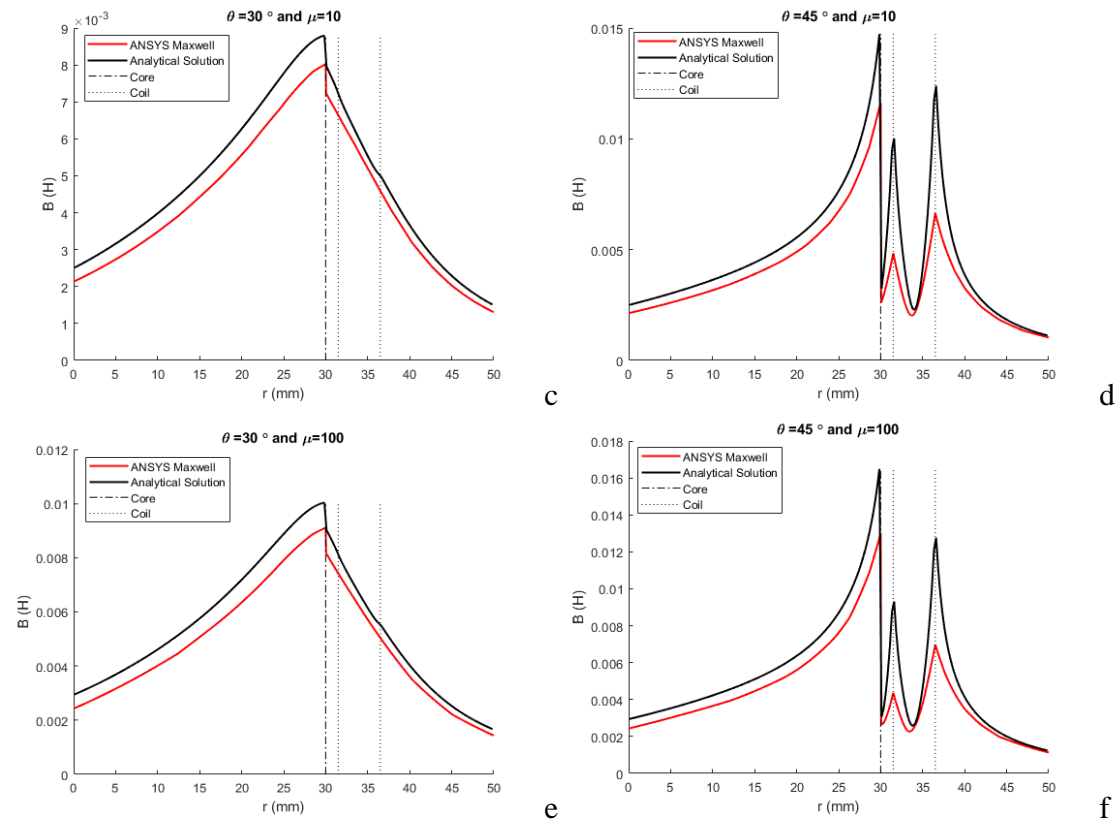

Figure 7

in the coordinate of $\theta=30^{\circ}$ for $\mu=1, \mu=10, \mu=100$, the comparison of the magnitudes of the magnetic field with the FEA results (a)(c)(e). In the coordinate of $\theta=45^{\circ}$ for $\mu=1, \mu=10, \mu=100$, the comparison of the magnitudes of the magnetic field with the FEA results $(b)(d)(f)$.

\section{Calculation of Inductance Coefficients}

\subsection{The Self-Inductance Coefficient}

In electromagnetic systems, the variation of the magnetic field causes an electric field. The voltage is induced on a ring-shaped copper wire that is exposed to a variable magnetic field. The Faraday Law gives the relationship between the current generated in the wire exposed to a variable magnetic field and the variation of the magnetic field [19]. If the connection between $\mathbf{E}$ and $\mathbf{A}$ is used, the voltage $\mathrm{V}$ in the wire can be calculated applying the magnitude of $\mathbf{A}$;

The voltage generated in the $\mathrm{N}$ coil winding; 
$V=-\frac{N}{S} \iint_{d S^{\prime}}\left\{\int_{d r} \mathbf{E} \square d \mathbf{r}\right\} d S^{\prime}$
$V=i \omega \frac{N}{S} \iint_{d S^{\prime}}\left\{\int_{d r} \mathbf{A} \square d \mathbf{r}\right\} d S^{\prime}$

The innermost integral is the voltage formed by a single-winding coil $\left(r_{0}, \theta_{0}\right)$ on itself; Here, $\mathbf{E}$ is taken as $E\left(r_{0}, \theta_{0}\right)$. This voltage is integrated on the cross-section and multiplied by the number of windings per unit area.

$$
\begin{aligned}
& V=i \omega \frac{N}{S} \iint_{\substack{\text { cross } \\
\text { section }}}\left\{A\left(r_{0}, \theta_{0}\right) r_{0} \sin \theta_{0} \int_{0}^{2 \pi} d \varphi\right\} d S \\
& V=i \omega \frac{N}{S} \iint_{\substack{\text { cross } \\
\text { section }}}\left\{A\left(r_{0}, \theta_{0}\right) r_{0} \sin \theta_{0} \int_{0}^{2 \pi} d \varphi\right\} r_{0} d r_{0} d \theta_{0}
\end{aligned}
$$

Where $\mathbf{A}, A^{(c)}, d S=r_{0} d r_{0} d \theta_{0}$ and $d r=r_{0} \sin \theta_{0} d \varphi$ on the cross-section, if the equation is arranged by substituting Eq. (24) with Eq. (21); then

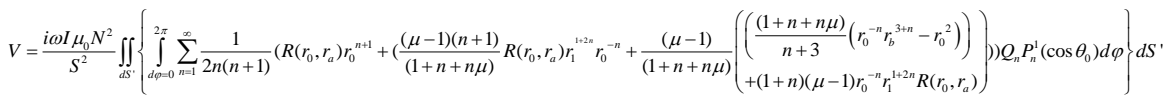

$$
\begin{aligned}
& V=\frac{\pi i \omega l \mu_{0} N^{2}}{S^{2}} \int_{d_{b}=r_{a}}^{n} \sum_{n=1}^{\infty} \frac{1}{n(n+1)} Q_{n}^{2}\left(R\left(r_{0}, r_{a}\right) r_{0}^{n+2}+\frac{(\mu-1)(n+1)}{(1+n+n \mu)} R\left(r_{0}, r_{a}\right) r_{1}^{1+2 n} r_{0}^{-n+1+1}+\left(\begin{array}{l}
\frac{(\mu-1)}{(n+3)}\left(\left(r_{0}^{-n+1} r_{b}^{3+n}-r_{0}^{4}\right)\right) \\
+\frac{1}{(1+n+n \mu)}(1+n) r_{0}^{-n+1} r_{1}^{1+2 n} R\left(r_{0}, r_{a}\right)
\end{array}\right)\right) d r_{0}
\end{aligned}
$$

Let $Q_{n}\left(\theta_{a}, \theta_{b}\right)=\int_{\theta_{a}}^{\theta_{b}} \sin \theta_{0} P_{n}^{1}\left(\cos \theta_{0}\right) d \theta_{0}$ and the $\mathrm{Z}$ is the impedance of the coil;

$$
\begin{aligned}
& \mathrm{Z}=\frac{\mathrm{V}}{\mathrm{I}}=\mathrm{i} \omega \mathrm{L} \\
& L=\frac{\pi \mu_{0} N^{2}}{S^{2}} \int_{d_{0}=r_{a}}^{n_{n}} \sum_{n=1}^{\infty} \frac{Q_{n}^{2}}{n(n+1)}\left(R\left(r_{0}, r_{a}\right) r_{0}^{n+2}+\frac{(\mu-1)(n+1)}{(1+n+n \mu)} R\left(r_{0}, r_{a}\right) r_{1}^{1+2 n} r_{0}^{-n+1}+\left(\begin{array}{l}
\frac{(\mu-1)}{(n+3)}\left(\left(r_{0}^{-n+1} r_{b}^{3+n}-r_{0}^{4}\right)\right) \\
+\frac{1}{(1+n+n \mu)}(1+n) r_{0}^{-n+1} r_{1}^{1+2 n} R\left(r_{0}, r_{a}\right)
\end{array}\right) d r_{0}\right.
\end{aligned}
$$

Eq. (27) gives the self-inductance coefficient of a multi-winding coil. Since the integral process cannot be calculated analytically, they are calculated numerically. 


\subsection{The Mutual Inductance Coefficients}

To calculate the mutual inductance coefficient, let's consider two concentric single-winding coils in spherical coordinates as seen in Figure 8a. When a variable current flows on coil 1, a variable magnetic field is generated in the air and magnetic core. An electromotive force (emf-voltage) is induced on Coil 2 due to the changing magnetic field. $\mathrm{M}_{\mathrm{ij}}$ is used to calculate the interaction between the two coils. Here, $\gamma$ is the angle between the axes of the coils, $\alpha$ and $\beta$ is the angular coordinate of the coils concerning their set of axes, $a$ and $b$ are the $r$ coordinates where the coils are located. $\varphi^{\prime}$ and $\theta^{\prime}$ are the angular coordinates concerning the fixed set of axes of the second coil. The representation of the coils as multi-windings is given in Figure $8 \mathrm{~b}$ and Figure 8c.

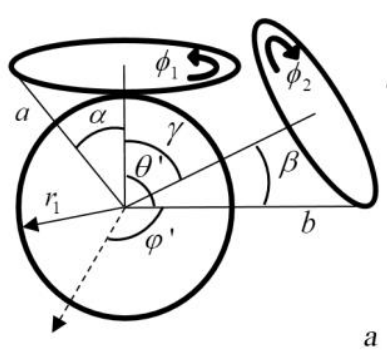

a

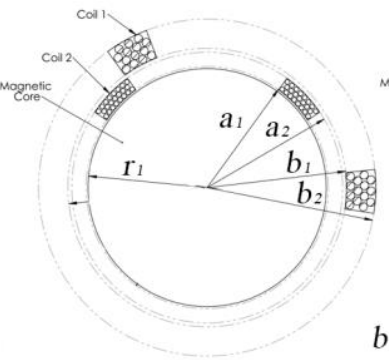

$b$

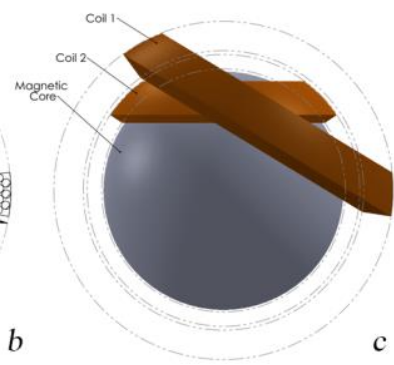

Figure 8

Two concentric single-winding coils (a), two concentric multi-winding coils (b), three-dimensional view (c)

If $\mathrm{a}<\mathrm{b}$ in the single-winding coil method, the mathematical form of the voltage that the first coil generates on the second coil is given in Eq. (23). The coil with the $\mathrm{N}_{1}$ number of windings and radius a is located in the $\propto$ position in spherical coordinates. The vector potential of $A_{i}^{(2)}\left(b, \theta^{\prime}\right)$ which the coil creates is given by Eq. (21).

$$
\begin{aligned}
& A_{i}^{(2)}\left(b, \theta^{\prime}\right)=\sum_{1}^{\infty} d_{n}^{i} b^{-n-1} P_{n}^{1}\left(\cos \theta^{\prime}\right) \\
& \left.d_{n}^{i}=\frac{N_{1}(\mu-1) I \mu_{0}}{S_{1} 2 n(n+1)(1+n+n \mu)}\left(\begin{array}{l}
\left(\frac{(1+n+n \mu)}{n+3}\left(a_{2}^{3+n}-a_{1}^{3+n}\right)\right) \\
+(1+n)(\mu-1) r_{1}^{1+2 n} R\left(a_{1}, a_{2}\right)
\end{array}\right)\right) \int_{\alpha_{1}}^{\alpha_{2}} \sin \alpha_{0} P_{n}^{1}\left(\cos \alpha_{0}\right) d \alpha_{0}
\end{aligned}
$$

Using Eq. (21) and Eq. (23), the mathematical form of the voltage expression generated in the second coil with the $\mathrm{N}_{2}$ number of windings and located in $b$ and $\beta$ coordinates is given below; 


$$
\begin{aligned}
& V=\frac{N_{2}}{S_{2}} \iint_{d S^{\prime}}\left\{\int_{d r} A \llbracket d \mathbf{r}\right\} d S^{\prime} \\
& V=i \omega \frac{N_{2}}{S_{2}} \iint_{d S^{\prime}}\left\{\int_{d \varphi}^{2 \pi} \sum_{n=1}^{\infty}\left(\begin{array}{l}
\left(\frac{(1+n+n \mu)}{n+3}\left(a_{2}^{3+n}-a_{1}^{3+n}\right)\right) \\
+(1+n)(\mu-1) r_{1}^{1+2 n} R\left(a_{1}, a_{2}\right)
\end{array}\right) b^{-n} P_{n}^{1}\left(\cos \theta^{\prime}\right) \sin \beta d \varphi\right\} d S^{\prime}
\end{aligned}
$$

Here, if $\mathrm{r}=\mathrm{b}$ and $d S^{\prime}=r d r d \beta$; then

$$
\begin{aligned}
& V=i \omega \sum_{n=1}^{\infty} \frac{\pi N_{2} N_{1}(\mu-1) I \mu_{0}}{S_{2} S_{1} n(n+1)(1+n+n \mu)} e_{n}\left\{\begin{array}{cc}
n=1, & b_{2}-b_{1} \\
n=2, & \ln \left(\frac{b_{2}}{b_{1}}\right) \\
n>2, & \frac{1}{2-n}\left(b_{2}^{2-n}-b_{1}^{2-n}\right)
\end{array}\right\} \beta \alpha P_{n}(\cos \gamma) \\
& e_{n}=\left(\begin{array}{l}
\left(\frac{(1+n+n \mu)}{n+3}\left(a_{2}^{3+n}-a_{1}^{3+n}\right)\right) \\
+(1+n)(\mu-1) r_{1}^{1+2 n} R\left(a_{1}, a_{2}\right)
\end{array}\right)
\end{aligned}
$$

$$
\begin{aligned}
& Z=\frac{V}{I}=i \omega M_{12} \\
& M_{12}=\sum_{n=1}^{\infty} \frac{\pi N_{2} N_{1}(\mu-1) \mu_{0}}{S_{2} S_{1} n(n+1)(1+n+n \mu)} e_{n}\left\{\begin{array}{lc}
n=1, & b_{2}-b_{1} \\
n=2, & \ln \left(\frac{b_{2}}{b_{1}}\right) \\
n>2, & \frac{1}{2-n}\left(b_{2}^{2-n}-b_{1}^{2-n}\right)
\end{array}\right\} \beta \alpha P_{n}(\cos \gamma)
\end{aligned}
$$

For a multi-winding coil, where $\mathrm{a}_{1}, \mathrm{a}_{2}, \mathrm{~b}_{1}$, and $\mathrm{b}_{2}$ are the coil radius, $\alpha_{1}, \alpha_{2}, \beta_{1}$ and $\beta_{2}$ are the angular coordinates concerning their set of axes, $\gamma$ is the angle between the coil axes, the mutual inductance coefficients $\left(M_{12}\right)$ of two coils is computed with Eq. (31) (Figure 8). Figure 9 shows how to program calculate $\mathrm{M}_{\mathrm{ij}}$ for each $\mathrm{P}_{\mathrm{i}}(\mathrm{r}, \theta)$,

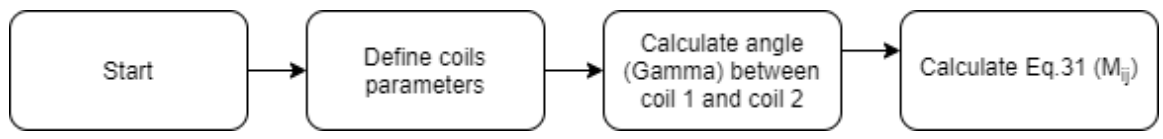

Figure 9

Mutual Inductance Coefficients 
The mutual inductance coefficient is of great importance in the time-dependent analysis of wireless energy transfer and mobile electrodynamic systems. Magnetic field magnitudes in the spherical coordinate system under the assumption of 2-dimensional axial symmetry can be calculated rapidly with the programs of ANSYS Maxwell, FEA, etc. However, if there is a second coil in the geometry that breaks the axial symmetry, it becomes necessary to design and calculate the models in 3 dimensions. In this case, the FEA program requires a long, timeconsuming analysis by using a large number of elements. The fact that the analytical equations are given in Eq. (31) can be calculated easily and quickly will provide the result in a short time at the preliminary design stage.

Table 2

The mutual inductance analysis parameters

\begin{tabular}{lll} 
Parameter & Coil 1 & Coil 2 \\
\hline $\mathrm{r}_{1}$ & $30 \mathrm{~mm}$ & \\
\hline $\mathrm{a}_{1}, \mathrm{~b}_{1}$ & $36.5 \mathrm{~mm}$ & $44 \mathrm{~mm}$ \\
\hline $\mathrm{a}_{2}, \mathrm{~b}_{2}$ & $41.5 \mathrm{~mm}$ & $49 \mathrm{~mm}$ \\
\hline$\alpha_{1}, \beta_{1}$ & $75^{\circ}$ & $75^{\circ}$ \\
\hline$\alpha_{2}, \beta_{2}$ & $105^{\circ}$ & $105^{\circ}$ \\
\hline$\gamma$ & $0^{\circ}$ & $0^{\circ} \leq \gamma \leq 360^{\circ}$ \\
\hline$\mu$ & $1,10,100,1000$ & \\
\hline$N_{1}, N_{2}$ & 180 & 150 \\
\hline $\mathrm{D} 1, \mathrm{D}_{2}$ & $0.75 \mathrm{~mm}$ & $0.5 \mathrm{~mm}$ \\
\hline $\mathrm{R} 1, \mathrm{R} 2$ & $3.35 \Omega$, & $2.6 \Omega$ \\
\hline $\mathrm{R} 3$ & $10.4 \Omega$ & \\
\hline
\end{tabular}

The results of the FEA program and analytical calculations by using the geometric properties given in Table 2 are presented in Figure 10. The problem parameters discussed in the experimental studies reviewed in Chapter 6 were also chosen the same values. It is seen that the mutual inductance results obtained from analytical calculations and the FEA model are compatible. While calculations take 60 minutes in the FEA model, calculations are completed in as little as 1 minute with analytical formulas using the suggested approach on the same computer. Therefore, the suggested approach can be prefered for the necessary calculations at the preliminary design stage rather than the FEA application. 

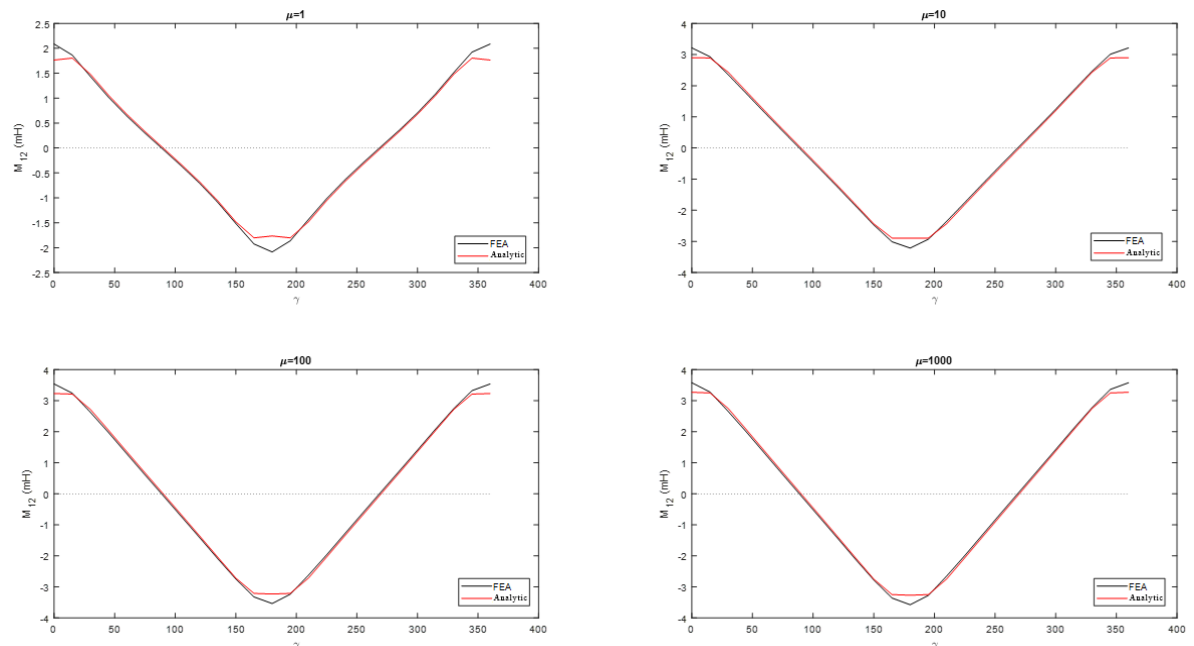

Figure 10

The comparison of the FEA and analytical calculations at different values of $\mu$

\section{Experimental Results}

In order to compare the analytical formulas found for mutual inductance and selfinductance coefficients with the experimental results, an experimental setup that can be produced with a 3D printer was designed (Figure 11). The experiment setup consists of a fixed coil (Coil 1), a moving coil (Coil 2), a magnetic core and carrier legs. The descriptions of the items of the experimental setup are given in Table 3. The design parameters are given in Table 2 .
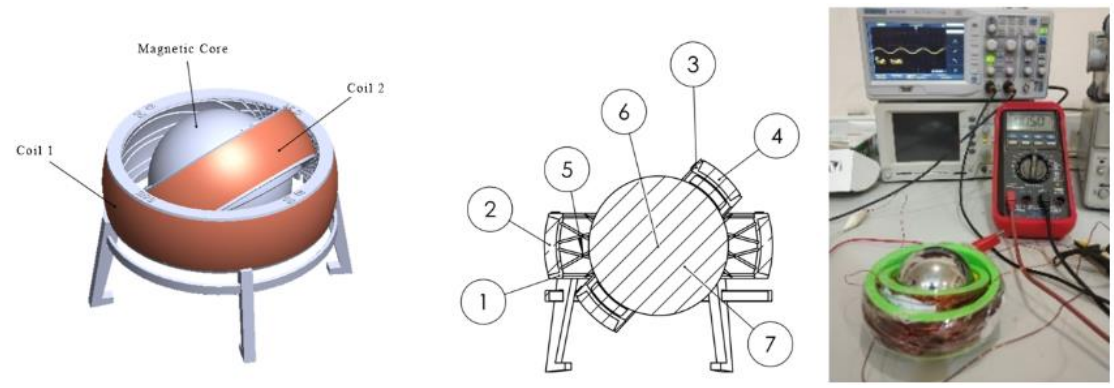

Figure 11

the Experiment Setup 
Table 3

The descriptions of the experimental items

\begin{tabular}{cl} 
Number & Description \\
\hline 1 & The body part on which the fixed coil is wound \\
\hline 2 & The windings of the fixed coil \\
\hline 3 & The body part on which the moving coil is wound \\
\hline 4 & The windings of the moving coil \\
\hline 6 & $\begin{array}{l}\text { Angular measuring lines marked at an angle of } 15^{\circ} \text { on the } \\
\text { fixed body to measure at different angles. }\end{array}$ \\
\hline 7 & The central rotation point \\
\hline
\end{tabular}

After the body parts are produced with the 3D printer, 180 turns of $\varnothing 0.75 \mathrm{~mm}$ diameter of copper wire are wound on the body (Coil 1), and 150 turns of $\varnothing 0.5$ $\mathrm{mm}$ diameter of copper wire are wound on the second body (Coil 2). By modeling the coil structures in the ANSYS Maxwell program, the self-inductance coefficients are compared with the experimental results in Table 4. It was observed that the values of the inductance coefficients in all three studies were quite compatible with each other. The devices and the models of which used in the experiment are given in Table 5.

Table 4

The comparison of self-inductance coefficients

\begin{tabular}{lll} 
Self-inductance & Coil 2 & Coil 1 \\
\hline Measurement & $4,26 \mathrm{mH}$ & $2,86 \mathrm{mH}$ \\
\hline The Maxwell program & $5,196 \mathrm{mH}$ & $3,729 \mathrm{mH}$ \\
\hline Analytical results & $4.662 \mathrm{mH}$ & $3.334 \mathrm{mH}$ \\
\hline
\end{tabular}

Table 5

The devices used in measurements and their features

\begin{tabular}{ll} 
Device Type & Brand/Model \\
\hline Oscilloscope & AA TECH/ADS-3072B Digital Storage Oscilloscope \\
\cline { 2 - 2 } & Gw INSTEK/GDS-1022 DSO \\
\hline Power Unit & Gw INSTEK/SFG-2107 Function Generator \\
\hline Inductance Meter & UNI-T/UT600 \\
\hline Multi-meters & BRYMEN BM510, FLUKE 106 \\
\hline
\end{tabular}

In the experiment, $\mathrm{L}_{\mathrm{ii}}$ was measured with the UNI-T measuring device. Since it was not possible to measure $\mathrm{M}_{\mathrm{ij}}$ directly, the current and voltage values in Coil 1 and Coil 2 were measured and calculated with Eq. (32) and Eq. (33).

$$
\begin{aligned}
& \mathrm{I}=\mathrm{I}_{\text {max }} \cos (\mathrm{wt}) \\
& e_{2}=M_{12} \frac{d I_{1}}{d t}
\end{aligned}
$$




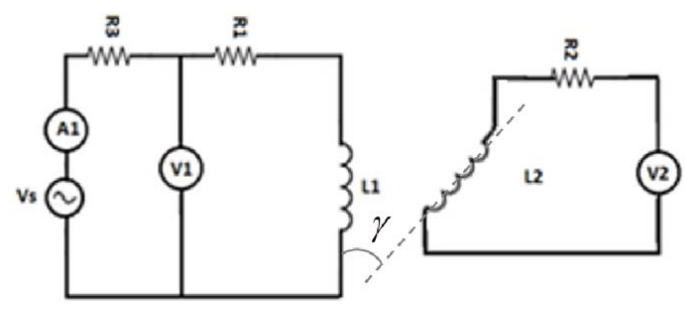

Figure 12

Electrical circuit diagram

Figure 12 shows the experimental setup circuit. The fixed coil is connected in series with a $50 \mathrm{~Hz}, 12 \mathrm{~V}$ of power supply with a $10.4 \Omega$ of resistance, and the current and voltage on the fixed coil were recorded with the voltmeter (V1) and ammeter (A1). R1 and R2 are due to coil wires. R3 is used for limiting current. The voltage value formed by connecting the moving coil leads to the voltmeter (V2) was recorded in the range of $0^{\circ}-360^{\circ}$ by changing the $\gamma$ angle at $15^{\circ}$ intervals.

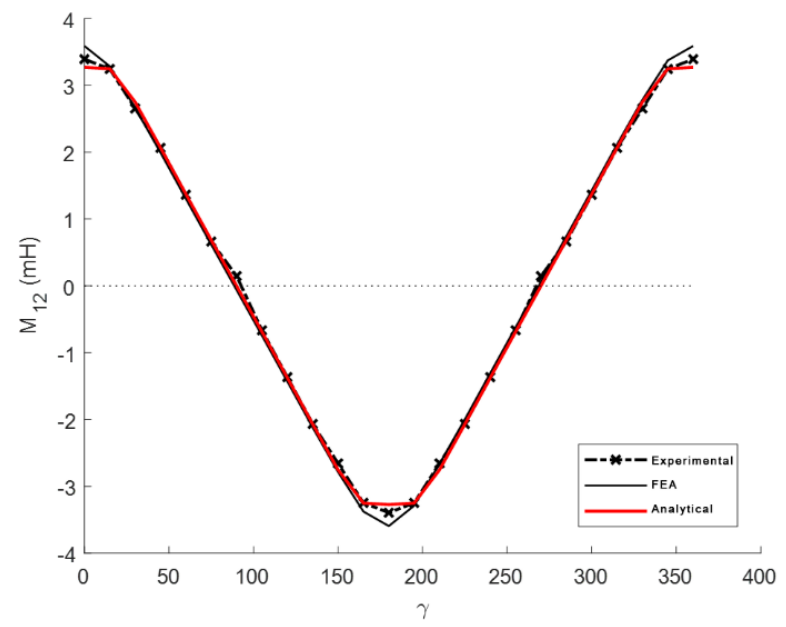

Figure 13

$\mathrm{M}_{12}$ at different $\gamma$ values, the comparison of experimental results, analytical results and FEA results

Based on the equivalent circuit approach from the gathered data, $\mathbf{M}_{12}$ was calculated using Eq. (31). The comparison of the data obtained from the experimental results with the analytical formulas and the FEA results is presented in Figure 13. It is evident that the analytical calculations made for $\mathrm{M}_{12}$ are consistent with the experimental results and the FEA results.

$$
R M S E=\sqrt{\frac{1}{D} \sum_{n=1}^{D}\left(y_{n}-\bar{y}_{n}\right)^{2}}
$$


Mean square error (MSE) [24] and root mean square error (RMSE) [25-27] are common methods use to compare the results of actual measurement values and calculation values. $D$ is the number of elements, $y_{n}$ is the measured values, and $\bar{y}_{n}$ is the located values. So, the analytical and FEA RMSE values are calculated by Eq. (34) of 0.0714 and 0.1073 , respectively.

\section{Conclusions}

In this study, the terms of $\mathbf{B}$ and $\mathbf{E}$ were calculated analytically by forming the geometry of a multi-winding coil with a magnetic core in spherical coordinates. Analytical formulas were derived for the multi-winding coil approach, given in Eq. (22). Since there are no existing analytical terms referring to the coils with the magnetic core in spherical coordinates, the obtained results were compared with the results of an air-core coil in spherical coordinates in the literature. The results were found to be consistent with the literature [22]. In order to view the effect of the magnetic sphere, different $\mu$ values and magnetic field distribution in different directions were examined. As a result of the analyses, it was observed that as the $\mu$ value increases, $\mathbf{B}$ accumulates towards the surface of the sphere (Figure 4). The coil structure with geometric properties is given in Table 1, the magnitudes of which $\mathbf{B}$ and $\mathbf{A}$ were obtained by using the formulas given in Eq. (22), and the FEA model was designed under the assumption of axial symmetry. When the results were examined, it was seen that the results of the analytical model and the FEA model were consistent (Figure 5 and Figure 6).

The experimental setup given in Figure 11 was created as the analytical and the FEA model. $\mathrm{L}_{\mathrm{ii}}$ for both coils in the experiment (Table 5) and $\mathrm{M}_{\mathrm{ij}}$ at different $\gamma$ angles were computed by analytical and FEA models. It was observed that the numerical results were consistent with the experimental results (Figure 13). When coils are intertwined, a small collapse is detected in the analytical solution. This collapse is caused by some of the windings that are in the reverse direction during the intertwining of the coil windings. However, the results gathered by analytical calculations are quite compatible with the experimental results. It takes 15 minutes on an average computer to create a three-dimensional FEA model and calculate the mutual inductance coefficient at an angle of $15^{\circ}$. The same results are measured in only 15 seconds using the developed analytical method. The obtained analytical results and their computation times are considered, it is seen that analytical results are useful. The FEA results are considered, despite the small deviations between the analytical solution and the experimental results is seen that the results obtained by the analytical method are acceptable. Considering the high cost of the FEA programs and long computation times in threedimensional models, the importance of using the suggested analytical solution is evident as a fast and free design tool in scientific studies.

\section{References}

[1] Conway, J. T. (2011) Mutual inductance for an explicitly finite number of turns. Progress In Electromagnetics Research B, 28, 273-287 
[2] Ravaud, R., Lemarquand, G., Lemarquand, V., Babic, S., \& Akyel, C. (2010) Mutual inductance and force exerted between thick coils. Progress In Electromagnetics Research, 102, 367-380

[3] Babic, S. I., \& Akyel, C. (2006) New analytic-numerical solutions for the mutual inductance of two coaxial circular coils with rectangular cross section in air. IEEE Transactions on Magnetics, 42(6), 1661-1669

[4] Conway, J. T. (2008) Noncoaxial inductance calculations without the vector potential for axisymmetric coils and planar coils. IEEE Transactions on Magnetics, 44(4), 453-462

[5] Conway, J. T. (2013) Analytical solutions for the self-and mutual inductances of concentric coplanar disk coils. IEEE transactions on magnetics, 49(3), 1135-1142

[6] Yang, T. T., \& Yang, J. J. (1975) The Effect of Cylindrical Ferromagnetic Shells on the Self and Mutual Inductance of Parallel Wires. IEEE Transactions on Electromagnetic Compatibility, (4), 234-237

[7] Babic, S. I., \& Akyel, C. (2008) Calculating mutual inductance between circular coils with inclined axes in air. IEEE Transactions on Magnetics, 44(7), 1743-1750

[8] Conway, J. T. (2012) Exact solutions for the mutual inductance of circular coils and elliptic coils. IEEE transactions on magnetics, 48(1), 81-94

[9] Lipinski, W., Rolicz, P., \& Sikora, R. (1975) Application of integral transforms to the analysis of the magnetic field of a spherical coil. IEEE Transactions on Magnetics, 11(5), 1552-1554

[10] Semenov, V. G. (1990) Synthesis of spherical methods of determining magnetic field source parameters of internal and external origin. Measurement Techniques, 33(12), 1236-1240

[11] Eaton, H. (1992) Electric field induced in a spherical volume conductor from arbitrary coils: application to magnetic stimulation and MEG. Medical and Biological Engineering and Computing, 30(4), 433-440

[12] Matute, E. A. (2005) On The Vector Solutions Of Maxwell Equations In Spherical Coordinate Systems, Rev. Mex. Fis. , Vol. E 51, 31-36

[13] Liu, C. Y., Andalib, T., Ostapchuk, D. C. M., \& Bidinosti, C. P. (2020) Analytic models of magnetically enclosed spherical and solenoidal coils. Nuclear Instruments and Methods in Physics Research Section A: Accelerators, Spectrometers, Detectors and Associated Equipment, 949, 162837

[14] Lee, K. M., Son, H., \& Joni, J. (2005, April) Concept development and design of a spherical wheel motor (SWM). In Proceedings of the 2005 
IEEE International Conference on Robotics and Automation (pp. 36523657) IEEE

[15] Dehez, B., Galary, G., Grenier, D., \& Raucent, B. (2006) Development of a spherical induction motor with two degrees of freedom. IEEE Transactions on Magnetics, 42(8), 2077-2089

[16] Fernandes, J. F., \& Branco, P. C. (2016) The shell-like spherical induction motor for low-speed traction: electromagnetic design, analysis, and experimental tests. IEEE transactions on industrial electronics, 63(7), 43254335

[17] Zhang, C., Yuan, L., Zhang, J., Chen, J., Chen, C. Y., Chen, S., \& Yang, G. (2018, April) Analytical models of electromagnetic field and torques in a novel reaction sphere actuator. In 2018 IEEE International Conference on Applied System Invention (ICASI) (pp. 271-274) IEEE

[18] Zhang, J., Yuan, L., Liao, Y., Zhang, C., Chen, C. Y., Chen, S., \& Yang, G. (2018, April) Torque optimization of a novel reaction sphere actuator based on support vector machines. In 2018 IEEE International Conference on Applied System Invention (ICASI) (pp. 263-266) IEEE

[19] Griffiths, D. J. (1998) Introduction to electrodynamics. New Jersey: Prentice Hall., 3th ed., ISBN 0-13-805326-X

[20] Jackson, J. D. (1962) Classical electrodynamics john wiley \& sons. Inc., New York, 13, Chapter 5, LCCCN:62-8774

[21] Paul, C. R. (2010) Inductance: loop and partial. John Wiley \& Sons. New Jersey A.B.D. Chapter 3, ISBN 978-0-470-46188-4

[22] Smythe, W. B. (1989) Static and dynamic electricity. Taylor \& Francis Publisher New York A.B.D., ISBN 0-89116-916-4

[23] Theodoulidis, T., \& Kriezis, E. E. (2006) Eddy Current Canonical Problems (with Applications to Nondestructive Evaluation) Tech Science Press. Forsyth, GA, USA., 978-0971788015

[24] Hedrea, E. L., Precup, R. E., Roman, R. C., \& Petriu, E. M. (2021) Tensor product-based model transformation approach to tower crane systems modeling. Asian Journal of Control., 23 (3), 1313-1323

[25] Precup, R. E., Teban, T. A., de Oliveira, T. E. A., \& Petriu, E. M. (2016, July) Evolving fuzzy models for myoelectric-based control of a prosthetic hand. In 2016 IEEE International Conference on Fuzzy Systems (FUZZIEEE) (pp. 72-77) IEEE

[26] Juang, C. F., Lin, Y. Y., \& Huang, R. B. (2011) Dynamic system modeling using a recurrent interval-valued fuzzy neural network and its hardware implementation. Fuzzy sets and systems, 179(1) 83-99 
[27] Precup, R. E., Teban, T. A., Albu, A., Borlea, A. B., Zamfirache, I. A., \& Petriu, E. M. (2019, June) Evolving fuzzy models for prosthetic hand myoelectric-based control using weighted recursive least squares algorithm for identification. In 2019 IEEE International Symposium on Robotic and Sensors Environments (ROSE) (pp. 164-169) IEEE 